\title{
Clinical and Dosimetric Factors Impacting Radiation Pneumonitis in Isotoxically Dose-Escalated NSCLC
}

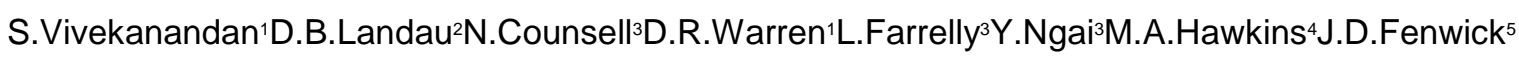

\section{Purpose/Objective(s)}

Radiation pneumonitis (RP) has been related to various clinical and radiation dosimetric factors such as mean lung equivalent dose in 2 Gy fractions (ML-EQD2). Here we test the hypothesis that other factors impact grade 2 or higher RP within 6 months of radiotherapy (RT) ( $\geq G 2 R P$ ) when ML-EQD2 is controlled via isotoxic dose-escalation.

\section{Materials/Methods}

We analyzed 114 patients treated to doses of 63-73 Gy in 30 fractions over 5 or 6 weeks in a prospective trial of isotoxically escalated RT with concurrent chemotherapy (CT). The predicted rate of $\geq G 2 R P$ was $20 \%$. An initial prescribed tumor dose (PD) was selected to achieve ML-EQD2 of 18.2 Gy. PD was reduced by $10 \%$ for CT toxicity, and further to meet other organ constraints. Dose volume histograms (DVHs) were extracted for these structures: Whole lung minus GTV (LGTV), lung on contralateral (CL) and ipsilateral (IL) sides of the tumor, CL and IL split into equal thirds (upper, middle, and lower sections). Ninety-five percent of the variance between L-GTV DVHs was represented by 7 principal components (PC) with eigenvalue $>1$. Maximum RP scores within 6 months of RT were collated. Univariable logistic regression analysis (UVA) was performed to ascertain associations of clinical factors, L-GTV PCs and ML-EQD2 with $\geq G 2 R P$ risk. Model 1 represents the best multivariable (MV) model obtained from these factors via stepwise selection using Akaike information criterion. The significant L-GTV PC in the MV model was replaced by the L-GTV and CL/IL section volumes irradiated to the dose levels represented by that PC for further localization.

\section{Results}

Mean PD was 67.1 Gy. ML-EQD2 mean was 14.4 Gy $(95 \% \mathrm{Cl}=13.9-14.9)$. The $\geq G 2 R P$ rate was $26 \%$. On UVA, there was no evidence that ML-EQD2 mean was associated with $\geq G 2 R P$ risk, but $\geq G 2 R P$ risk increased with poor $P S$ and high L-GTV PC5 (representing greater L-GTV volumes receiving 6-9 Gy, L-GTV 6-9). L-GTV PC5, poor PS, and high baseline FVC (FVC0) were significantly associated with $\geq G 2 R P$ 
risk (MV Model 1). L-GTV 6 -9 and CL lower segment (CLL) receiving 6-9 Gy (CLL6-9) were significantly associated with $\geq G 2 R P$ risk when they replace L-GTV PC5 (MV model 2 and 3).

\section{Conclusion}

ML-EQD2 mean is a poor predictor for $\geq G 2 R P$ in these isotoxically escalated patients, amongst whom there was limited lung mean dose variability. Poor PS, high FVC0 and greater lung volumes receiving 6-9 Gy were associated with increased $\geq \mathrm{G} 2 \mathrm{RP}$ risk.

Abstract 329; Table 1.

\begin{tabular}{|c|c|c|c|c|}
\hline \multirow[t]{2}{*}{ Variables } & \multirow{2}{*}{$\begin{array}{c}\text { UVA } \\
\text { OR }(95 \% \text { CI }) P \text {-value }\end{array}$} & \multicolumn{3}{|c|}{ MVA } \\
\hline & & OR $(95 \%$ CI $) P$-value & OR $(95 \%$ CI $) P$-value & OR $(95 \%$ CI $) P$-value \\
\hline PS 1 Vs 0 & $\begin{array}{l}2.447(1.005-5.961) \\
0.049\end{array}$ & $\begin{array}{l}3.716(1.370-10.084) \\
.010\end{array}$ & $\begin{array}{l}3.433(1.298-9.078) \\
.013\end{array}$ & $\begin{array}{l}3.484(1.316-9.224) \\
.012\end{array}$ \\
\hline PD & $\begin{array}{l}1.064(0.946-1.197) \\
0.298\end{array}$ & - & - & - \\
\hline PTV & $\begin{array}{l}1.001(0.999-1.003) \\
0.511\end{array}$ & - & - & - \\
\hline $\begin{array}{l}\text { ML- } \\
\text { EQD2mean }\end{array}$ & $\begin{array}{l}0.961(0.823-1.123) \\
0.617\end{array}$ & - & - & - \\
\hline FVC0 & $\begin{array}{l}1.018(0.885-1.042) \\
0.115\end{array}$ & $\begin{array}{l}1.025(1.000-1.050) \\
.049\end{array}$ & $\begin{array}{l}1.026(1.001-1.051) \\
.038\end{array}$ & $\begin{array}{l}1.023(.998-1.048) \\
.067\end{array}$ \\
\hline L-GTV PC5 & $\begin{array}{l}1.578(1.050-2.373) \\
.028\end{array}$ & $\begin{array}{l}1.738(1.138-2.654) \\
.010\end{array}$ & $\mathrm{~N} / \mathrm{C}$ & $\mathrm{N} / \mathrm{C}$ \\
\hline $\mathrm{L}_{-\mathrm{GTV}_{6-9}}$ & $\begin{array}{l}1.089(.999-1.188) \\
.053\end{array}$ & $\mathrm{~N} / \mathrm{C}$ & $\begin{array}{l}1.114(1.014-1.223) \\
.025\end{array}$ & $\mathrm{~N} / \mathrm{C}$ \\
\hline $\mathrm{CLL}_{6-9}$ & $\begin{array}{l}1.053(1.001-1.107) \\
.048\end{array}$ & $\mathrm{~N} / \mathrm{C}$ & $\mathrm{N} / \mathrm{C}$ & $\begin{array}{l}1.060(1.004-1.119) \\
.036\end{array}$ \\
\hline
\end{tabular}

$\mathrm{N} / \mathrm{C}=$ not considered

Author Disclosure:S. Vivekanandan: None. D.B. Landau: None. N. Counsell: None. D.R.

Warren: None. L. Farrelly: None. Y. Ngai: None. M.A. Hawkins: None. J.D. Fenwick: None. 